\title{
Train Young Colonoscopist with Sigmoidoscopy - Performance, Adenoma Detection Rate and More
}

\section{Liu Wenfang, Cao Longyang, Zhao Yaying, Chen Xinglin and Ouyang Hong*}

The Affilated, Lin'an Hospital of Zhejiang Medical College, China

*Corresponding Author: Hong Ouyang, The Affilated, Lin'an Hospital of Zhejiang

Medical College, China. Email: max.ouyang@gmail.com
Received: September 27, 2021

Published: October 18, 2021

(C) All rights are reserved by Ouyang Hong., et al.

\begin{abstract}
Objective: To observe polyp/adenoma detection rate of sigmoidoscopy among doctors with different skill level.

Method: 5 doctors examined healthy checkups respectively. Upon detection, diagnosis adenoma/non-adenoma according to Kudo classification. Recommend total colonoscopy (TC) resection for adenomas.

Results: 1666 healthy checkups examined. 201 resection recommendations were made, and 120 actually received TC adenoma resection. All doctors finish sigmoidoscopy successfully, with different intubation depth. Intubation depth is highly related with prior finished TC number of the doctor. R value is 0.7593 for reaching descending, 0.9416 for transcending, 0.9786 for ascending colon. Total polyp detection rate $32.05 \%$, adenoma detection rate (ADR) $12.06 \%$. ADR vary significantly among doctors, from $9.39 \%$ to $17.36 \%$. ADR also highly related with prior finished TC number, $\mathrm{R}=0.8234$. Adenoma diagnosis was verified with histology, from $72.73 \%$ to $91.67 \%$. There is no difference among doctors, $\mathrm{p}=0.9716$. Total adenoma missing rate of sigmoidoscopy is $17.5 \%$. There is no difference among doctors, $\mathrm{p}=0.3898$.

Conclusions: The ADR of sigmoidoscopy is fare. Skill level has important influence to ADR of sigmoidoscopy, but not diagnosis accuracy.
\end{abstract}

Keywords: Healthy Checkup; Sigmoidoscopy; Adenoma Detection Rate; Kudo Classification

\section{Foreword}

Colorectal cancer is one of the leading causes of cancer-related death globally. It starts as a noncancerous polyp that grows slowly and then becomes cancerous. When it complete the transformation and cause symptom, it is often incurable. So western societies developed screening projects for un-symptomatic people, targeting on find and remove polyps, with endoscopy. These guidelines and project made huge success achieving decline in both incidence rate and mortality rate of the cancer.

For anyone seeking replicate this success, he must face the hardship of training doctors, adequate in number, and competence in capability. The challenge is, how to put the expensive scope (maybe comparable to annual income of a junior doctor) on trainee's hand, without causing too much stress. Make them focus on diagnose, and serve patients right.

A less demanding examination could be the answer.

Sigmoidoscopy (SC) is a recommended colon cancer screening tool [1-5]. Like total colonoscopy (TC), it examines colon mucosa to find pre-cancerous lesions such as adenoma. Adenoma detection rate (ADR) is the most important quality indicator for both colonoscopy and sigmoidoscopy. It relate closely with interval cancer, impacts clinical outcome [6,7]. We start offering sigmoidoscopy to healthy checkups since 2013. It is carryout mainly by young phy- 
sicians. To assure quality, we analyses polyps/adenoma detection of sigmoidoscopy and following total colonoscopy, as well as histology. We also analyses the performance indicator like intubation depth, comparing doctors with difference previous TC volume (as indicator of their skill level).

\section{Methods}

- Bowl preparation: Healthy checkups is asked to make a bowl movement at home before came to endoscopy unit in the morning. Lavation is made with sodium phosphate prior examination. Sigmoidoscopy is started after 1 or 2 extra bowl movement.

- Equipment: Conventional high definition flexible colonoscopy is used.

- Examiner: There are 5 colonoscopist. Dr. Ouyang hong as coach. The other 4 has passed basic training with previous success TC case ranging from 115-372. Each doctor responsible to a specific sigmoidoscopy morning shift. Typically, they need to finish 15-20 SC exam in 3 hours.

- Examine procedure: Without sedation, intubate as colonoscopy until reaching dirty lumen or patient indicate intolerance. If sigmoid cannot visualized because of stool, repeat lavation.

- Intubation depth: By observing lumen and touching patient's abdomen, intubation depth are determined by en- doscopist. Record as reaching ascending colon, transcending colon, descending colon and sigmoid colon.

- Diagnosis: Upon detecting polyps, the depth, shape and size is recorded. Pit pattern is carefully examined by approaching the lesion. Kudo classification is used to predict histology.

- Recommendation and follow up procedure: For polyps with kudo classification as possible adenoma, recommend TC polypectomy. Other patients with polyp, recommend TC examination. TC polypectomy and examination are conducted by one experienced endoscopist. Polyps found upon TC examination over $0.3 \mathrm{~cm}$ are also removed.

\section{Results}

From Aug 2013 to Oct 2014, 1666 sigmoidoscopy examines are made by 5 doctors. 201 TC polypectomy recommendation are made to patients. Among which 120 actually come and receive polypectomy.

169 polyps are resected, 132 proved adenoma by histology, 18 proved hyperplastic polyp. 94 patients proved to have adenoma, 58 found adenoma in dipper part colon which was not intubated by prior sigmoidoscopy. 21 found more adenoma in colon lumen which was intubated before. 1 patient cannot relocate the polyp found by sigmoidoscopy.

The results is listed as following.

\begin{tabular}{|l|c|c|c|c|c|c|}
\hline \multirow{2}{*}{ Doctor } & \multirow{2}{*}{ TC experience* } & \multirow{2}{*}{ SC Number } & \multicolumn{4}{|c|}{ Intubation depth } \\
\cline { 4 - 7 } & & & Sigmoid (\%) & Descending (\%) & $\begin{array}{c}\text { Transcending } \\
\text { (\%) }\end{array}$ & $\begin{array}{c}\text { Ascending } \\
\text { (\%) }\end{array}$ \\
\hline A Ouyang & 2781 & 409 & $89(21.76)$ & $176(43.03)$ & $118(28.85)$ & $26(6.36)$ \\
\hline B Cao & 372 & 390 & $256(65.64)$ & $109(27.95)$ & $18(4.62)$ & $7(1.79)$ \\
\hline C Zhao & 269 & 251 & $80(31.87)$ & $120(47.81)$ & $51(20.32)$ & $0(0.00)$ \\
\hline D Chen & 247 & 181 & $105(58.01)$ & $46(25.41)$ & $29(16.02)$ & $1(0.55)$ \\
\hline E Liu & 115 & 435 & $310(71.26)$ & $114(26.21)$ & $11(2.53)$ & $0(0.00)$ \\
\hline Total & - & 1666 & $840(50.42)$ & $565(33.91)$ & $227(13.63)$ & $34(2.04)$ \\
\hline
\end{tabular}

Table 1: Intubation depth of sigmoidoscopy and doctor's colonoscopy experience.

* Number of finished TC before this project.

Chi-square test, intubation depth differ significantly among doctors, $\mathrm{p}=0.0000$. Difference among 4 young doctors is also significant, $\mathrm{p}=0.0000$.

TC experience is highly associated with intubation beyond sigmoid. R values are, 0.7592766 for descending colon, 0.9415732 for transcending colon, 0.9786237 for ascending colon. 


\begin{tabular}{|l|c|c|c|c|}
\hline Doctor & $\mathbf{n}$ & $\begin{array}{c}\text { Non-adenoma } \\
(\%)\end{array}$ & Adenoma (\%) & Total (\%) \\
\hline A Ouyang & 409 & $87(21.27)$ & $71(17.36)$ & $158(38.63)$ \\
\hline B Cao & 390 & $73(18.72)$ & $40(10.26)$ & $113(28.97)$ \\
\hline C Zhao & 251 & $26(10.36)$ & $27(10.76)$ & $53(21.12)$ \\
\hline D Chen & 181 & $46(25.41)$ & $17(9.39)$ & $63(34.81)$ \\
\hline E Liu & 435 & $101(23.22)$ & $46(10.57)$ & $147(33.79)$ \\
\hline Total & 1666 & $333(19.99)$ & $201(12.06)$ & $534(32.05)$ \\
\hline
\end{tabular}

Table 2: Polyp/adenoma detection rate of sigmoidoscopy.

Chi-square test, There are significant differences in the discovery rate of endoscopic polyps and adenomas among doctors, $\mathrm{p}=$ $0.0000(7.576 \mathrm{e}-06)$; Eliminating senior doctors, $\mathrm{p}=0.001347$.

The discovery rate of adenomas of each doctor is highly correlated with past colonoscopy volume, $\mathrm{R}=0.8234129$; The discovery rate of non-adenomatous polyps is positively correlated with the number of past colonoscopy, $\mathrm{R}=0.3401347$, The total discovery rate is also related to past colonoscopy, $\mathrm{R}=0.7752555$.

\begin{tabular}{|l|c|c|c|c|}
\hline Doctor & n & Confirm (\%) & $\begin{array}{c}\text { New detection } \\
\text { (\%)* }\end{array}$ & Missing (\%)** \\
\hline A Ouyang & 37 & $28(75.68)$ & $18(48.65)$ & $5(13.51)$ \\
\hline B Cao & 31 & $23(74.19)$ & $14(45.16)$ & $6(19.35)$ \\
\hline C Zhao & 20 & $18(90.00)$ & $12(60.00)$ & $6(30.00)$ \\
\hline D Chen & 5 & $4(80.00)$ & $4(80.00)$ & $2(40.00)$ \\
\hline E Liu & 27 & $21(77.78)$ & $10(37.04)$ & $2(7.41)$ \\
\hline Total & 120 & $94(78.33)$ & $58(48.33)$ & $21(17.50)$ \\
\hline
\end{tabular}

Table 3: Subsequent colonoscopy treatment status (statistics of the number of patients).

* New findings that are not within the reach of the last inspection.

**New polyp found within the reach of the last inspection.

Chi-square test, There was no significant difference in the rate of new adenoma detection among doctors. $\mathrm{p}=0.3483$.

\section{Discussion}

With the changes in diet, the incidence of colorectal cancer in my country has increased significantly, and some areas have reached or exceeded the previous level in Western countries [8]. while Western countries which traditionally high in colorectal cancer incidence have reduced the incidence through systematic

\begin{tabular}{|l|c|c|c|c|c|}
\hline Doctor & $\mathbf{n}$ & $\begin{array}{c}\text { Found total } \\
(\%)\end{array}$ & $\begin{array}{c}\text { Adenomas } \\
\text { under } \\
\text { sigmoid- } \\
\text { oscopy }\end{array}$ & $\begin{array}{c}\text { New } \\
\text { discoveries } \\
\text { under TC* }\end{array}$ & $\begin{array}{c}\text { Missing } \\
\mathbf{( \% )}\end{array}$ \\
\hline A Ouyang & 409 & $158(38.63)$ & 50 & 30 & $6(10.71)$ \\
\hline B Cao & 390 & $113(28.97)$ & 42 & 10 & $7(14.29)$ \\
\hline C Zhao & 251 & $53(21.12)$ & 24 & 7 & $7(22.58)$ \\
\hline D Chen & 181 & $63(34.81)$ & 9 & 7 & $2(18.18)$ \\
\hline E Liu & 435 & $147(33.79)$ & 44 & 40 & $3(6.38)$ \\
\hline Total & 1666 & $534(32.05)$ & 169 & 94 & $25(12.89)$ \\
\hline
\end{tabular}

Table 4: Missing rate of sigmoidoscopy (Statistics on the number of polyps).

*The number of new finding in the following TC within the reach of the sigmoidoscopy, those beyond reach of first sigmoidoscopy is not calculated.

Chi-square test, There was no significant difference in adenoma missing among doctors. $\mathrm{p}=0.3898$.

\begin{tabular}{|l|c|c|c|c|c|c|}
\hline Doctor & n & n TC & $\begin{array}{c}\mathbf{n} \\
\text { adeno- } \\
\text { ma }\end{array}$ & $\begin{array}{c}\text { Pathology } \\
\text { confirmed } \\
(\%)\end{array}$ & $\begin{array}{c}\text { Hyperplastic } \\
\text { polyps (\%) }\end{array}$ & Total \% \\
\hline $\begin{array}{l}\text { A Ouy- } \\
\text { ang }\end{array}$ & 409 & 37 & 50 & $37(74.00)$ & $8(16.00)$ & $90.00 \%$ \\
\hline B Cao & 390 & 31 & 42 & $33(78.57)$ & $1(2.38)$ & $80.95 \%$ \\
\hline C Zhao & 251 & 20 & 24 & $22(91.67)$ & $1(4.17)$ & $95.83 \%$ \\
\hline D Chen & 181 & 5 & 9 & $8(88.89)$ & $0(0.00)$ & $88.89 \%$ \\
\hline E Liu & 435 & 27 & 44 & $32(72.73)$ & $8(18.18)$ & $90.91 \%$ \\
\hline Total & 1666 & 120 & 169 & $132(78.11)$ & $18(10.65)$ & $88.76 \%$ \\
\hline
\end{tabular}

Table 5: Pathological coincidental rate of sigmoidoscopy prediction.

Chi-square test, There was no significant difference in Pathological coincidental rate among doctors, $p=0.9716 \varangle$ Total $\%$ no significant difference, $\mathrm{p}=0.9932$.

colorectal cancer screening. According to the acting prevention guidelines, high-sensitivity fecal occult blood, sigmoidoscopy, and full colonoscopy are recommended as the main screening methods, and full colonoscopy is used as a further examination method whenever abnormalities are found. The latter two can directly observe the intestinal lumen and find precancerous changes-colonic 
adenoma $[1-5,9]$. It has proven that the efficacy of colonoscopy is better than that of fecal occult blood, but there are problems such as large resource consumption and complicated operation, which is difficult to adopt in our vast population.

Sigmoidoscopy is an important method, but rarely adopted in our country, especially as a physical examination item. Most healthy checkup plan will provide with stool OB test or rectal digit examination, some will provide rigid tube rectal endoscopy, and a small number of units will choose to provide full colonoscopy. The preparation of TC is complicated, time-consuming, and difficult to promote. Even patients with positive fecal occult blood find it difficult to make up their minds to undergo the examination.

There are randomized controlled studies confirmed that the application of sigmoidoscopy screening can significantly reduce the incidence of colon cancer. About 100,000 people participated in the study. The median observation period was 10.9 years. There were 253 cases of colorectal cancer and 77 deaths in the screening group; 1086 cases of colorectal cancer and 330 deaths in the control group. Fully demonstrated the efficacy of sigmoidoscopy in the prevention of colorectal cancer [10].

Compared with total colonoscopy, sigmoid colonoscopy has obvious advantages for healthy checkups. Patients do not need to undergo a complicated bowel preparation process, and most subjects can empty the left colon after enema and two bowel movements. In our practice for more than a year, we rarely found inadequate enema preparations, and no patients could not tolerate reaching the upper sigmoid colon. Some even achieve cecal intubation.

The participating doctors have different levels of operation, ranging from senior doctors who have completed tens of thousands of endoscopy to total newbie who have just passed basic training. However, every doctor is competent for the job. Epically for young doctors, they feel much less pressure, as it does not require reaching the ileocecal area.

In fact, there are significant differences in the insertion depth of the sigmoidoscopy between doctors with different skill levels. The depth of intubation is highly positively correlated with the number of colonoscopy accomplished in the past. the more, the deeper. Upon examination, when the intestinal cavity became dirty, we withdraw; the subject indicates intolerance, we withdraw. The latter one is only related to the looping of the endoscope, which is the indicator of operational proficiency. Therefore, this also shows that intestinal cleanliness is not the main factor that affects the intuba- tion depth of the flexible sigmoidoscopy. Most patients does have good left colon cleanliness after enema and defecation.

In this study, the total polyp discovery rate of sigmoidoscopy was $32.05 \%$, of which the adenoma discovery rate was $12.06 \%$. There are differences in polyps and adenomas detection among doctors. The lowest discovery rate was $9.39 \%$, and the highest was $17.36 \%$, respectively. The adenoma detection rates of the four young doctors were all around 10\%. There are reports about polyp/ adenoma detection rate of 93 examiners, the median polyp detection rate was $24.4 \%$, and the adenoma detection rate was $12.2 \%$, which was comparable to ours [7].

Adenoma detection rate and total detection rate are highly positively correlated with the number of previous endoscopic operations, while the detection rate of non-adenomatous polyps is weakly positive correlated. In the study of total colonoscopy, the most important factors affecting the adenomas detection rate are the bow prep, the withdrawal time, and the doctor [11]. Since the insertion depth (also the observation range) of sigmoidoscopy is highly related to the operation ability, and the observation time also affected by the observation range. This phenomenon is not surprising.

For this year, we have recommended a total of 201 patients to have polyps removed, and 120 of them came for treatment. Among these patients, $48.33 \%$ found new adenomas in the range not reached in the previous examination, and $17.50 \%$ found missing adenomas in the range reached in the previous examination. The adenoma missed rate of sigmoidoscopy is lower than previously reported $20 \%[12,13]$.

With the improvement of endoscope definition and the in-depth study of mucosal surface structure, the ability of endoscopic diagnosis has greatly improved [14]. Most experts emphasize the application of surface pattern classification during colonoscopy, determine whether endoscopic intervention is required. We trained the participating doctors with surface pattern classification. Require them to apply during sigmoidoscopy. and recommended whether to remove the polyp with the theory. The pathological results confirmed most of the diagnosis of adenomas under the microscope, and the accuracy of endoscopic diagnosis is $72.73-91.67 \%$. The diagnostic accuracy of junior doctors is not low, and there is no statistical difference compared with senior doctors.

Colon cancer is closely related to colorectal adenoma. There was indifference among Malignant potential of Hyperplastic polyps 
[15]. Like Hyperplastic polyps, HP, Sessile serrated polyp, SSA/P Traditional serrated adenoma, TSA. Pathologist may have difficulty differentiating. In this study, there was no statistical difference between the endoscopists, whether the diagnosis confirmation rate of simple adenoma or the total diagnosis confirmation rate after accounting for hyperplastic polyps. If hyperplastic polyps are included as adenoma, the adenoma confirmation rate rises to 88.8995.83\%.

\section{Conclusion}

Our practice has proved that the ability of sigmoidoscopy to detect colon adenomas is stable. The resources consumption are relatively low. In particular, it is less skill demanding. Junior doctors can quickly became competent after brief training. The examinee does not need complicated bowel preparations, so it may be able to scale-up to lager centralized examination. It could be a good ladder helping young doctors before they gain full power of colon intubation. Training like this can unleash there diagnosing potential with pattern classification.

\section{Bibliography}

1. Winawer SJ., et al. "Cascade colorectal cancer screening guidelines: a global conceptual model”. Journal of Clinical Gastroenterology 45.4 (2011): 297-300.

2. Jover R., et al. "Clinical practice guidelines: quality of colonoscopy in colorectal cancer screening”. Endoscopy 44.4 (2012): 444-451.

3. Qaseem A., et al. "Screening for colorectal cancer: a guidance statement from the American College of Physicians". Annals of Internal Medicine 156.5 (2012): 378-386.

4. U.S. Preventive Services Task Force. "Screening for colorectal cancer: recommendation and rationale". Annals of Internal Medicine 137.2 (2002): 129-131.

5. U.S. Preventive Services Task Force. "Screening for colorectal cancer: U.S. Preventive Services Task Force recommendation statement". Annals of Internal Medicine 149.9 (2008): 627-637.

6. Rex DK., et al. "Quality indicators for colonoscopy". The American Journal of Gastroenterology 101.4 (2006): 873-885.

7. Rogal SS., et al. "Relationship between Detection of Adenomas by Flexible Sigmoidoscopy and Interval Distal Colorectal Cancer". Clinical Gastroenterology and Hepatology 11.1 (2013): 7378.
8. Zheng Jingquan., et al. "Epidemiological study on the incidence of colorectal cancer in Pudong New Area, Shanghai". Chronic Disease Prevention and Control in China 11.3 (2003): 110-135.

9. Levin B., et al. "Screening and surveillance for the early detection of colorectal cancer and adenomatous polyps, 2008: a joint guideline from the American Cancer Society, the US Multi-Society Task Force on Colorectal Cancer, and the American College of Radiology". CA: A Cancer Journal for Clinicians 58.3 (2008): 130-160.

10. Holme Ø., et al. "Effect of flexible sigmoidoscopy screening on colorectal cancer incidence and mortality: a randomized clinical trial". JAMA 312.6 (2014): 606-615.

11. Chen SC and Rex DK. "Endoscopist can be more powerful than age and male gender in predicting adenoma detection at colonoscopy". The American Journal of Gastroenterology 102.4 (2007): 856-861.

12. Schoenfeld P., et al. "Accuracy of polyp detection by gastroenterologists and nurse endoscopists during flexible sigmoidoscopy: a randomized trial". Gastroenterology 117.2 (1999): 312-318.

13. Huang Yinglong., et al. "The missed diagnosis rate and risk factors of colorectal adenoma in colonoscopy". Chinese Journal of Digestive Endoscopy 27.6 (2010): 281-286.

14. Bruno M. "Magnification endoscopy, high resolution endoscopy, and chromoscopy; towards a better optical diagnosis". Gut 52 (2003): iv7-11.

15. Rex DK., et al. "Serrated Lesions of the Colorectum: Review and Recommendations From an Expert Panel". The American Journal of Gastroenterology 107.9 (2012): 1315-1330.

Volume 5 Issue 11 November 2021 (C) All rights are reserved by Hong Ouyang.,et al. 